\title{
Evaluation of the effects of sound exposure and low field electromagnetism on growth and antibiotics susceptibility of some microorganisms
}

\author{
Emmanuel Oluwaseun Garuba ${ }^{1^{*}} \mathbb{D}$, Obinna Markraphael Ajunwa ${ }^{2}$ and Aishat Nana Ibrahim-King ${ }^{1}$
}

\begin{abstract}
Background: The emergence of antibiotic-resistant microorganisms has been largely associated with drug misuse, drug abuse, and indiscriminate disposal of drugs; however, the interactions between some environmental factors and antibiotic resistance by microorganisms have been understudied. In this study, the effects of sound and electromagnetic field on the growth and antibiotic susceptibility of selected microorganisms to antibiotics were investigated.

Results: Microorganisms used include Gluconobacter oxydans ATCC 19,357, Rhodobacter sphaeroides ATCC 17,023, Citrobacter freundii ATCC 33,128, Yersina pestis ATCC 11,953, Bacillus subtilis 6633, Acetobacter aceti ATCC 15,973, Escherichia coli ATCC 25,922, Pseudomonas aeuriginosa ATCC 9027, Streptococcus pyogenes ATCC 19,613, Klebsiella pneumonia ATCC 25,955, Staphylococcus aureus ATCC 25,923 and Serratia marcescens ATCC 14,766. The antibiotics used were: Ciprofloxacin $5 \mu \mathrm{g}$, Imipenem $10 \mu \mathrm{g}$, Ampicillin $10 \mu \mathrm{g}$, Ceftazidime $30 \mu \mathrm{g}$ and Tetracycline $30 \mu \mathrm{g}$ for Gram-negative bacteria while Pefloxacin $10 \mu \mathrm{g}$, Gentamycin $10 \mu \mathrm{g}$, Amplicillin + Cloxacillin $30 \mu \mathrm{g}$, Cefuroxime $20 \mu \mathrm{g}$, Amoxacillin $30 \mu \mathrm{g}$, Ceftriaxone $25 \mu \mathrm{g}$, Ciprofloxacin $10 \mu \mathrm{g}$, Streptomycin $30 \mu \mathrm{g}$, Co-trimoxazole $30 \mu \mathrm{g}$, and Erythromycin $10 \mu \mathrm{g}$ for Grampositive bacteria, respectively. Acoustic treatment had varying effects on the antibiotics susceptibility profile of all test bacterial culture. Before exposure, P. aeruginosa had the highest zone of inhibition of $34 \pm 3.4 \mathrm{~mm}$, while B. subtilis had least inhibition zone of $12 \pm 2.8$. After exposure to acoustic treatment at $5000 \mathrm{~Hz} / 90 \mathrm{~dB}$ at $72 \mathrm{~h}, \mathrm{C}$. freundii had highest zone of inhibition of $32 \pm 0.7 \mathrm{~mm}$ and the least zone of $11 \pm 1.4 \mathrm{~mm}$ observed in P. aeruginosa. At $1125 \mathrm{~Hz} / 80 \mathrm{~dB}$ after $72 \mathrm{~h}, R$. sphaeroides had highest zone of $34 \pm 0.7 \mathrm{~mm}$ while $A$. aceti had least zone of inhibition of $10 \pm 0 \mathrm{~mm}$. Effect of electromagnetic flux treatment of $15 \mathrm{~min}$ showed $E$. coli to be the most inhibited having a growth rate of $0.08 \mathrm{log} \mathrm{cfu} /$ $\mathrm{mL}$, antibiotics testing showed $\mathrm{G}$. oxydans to have the highest zone of inhibition of $28 \pm 3.5 \mathrm{~mm}$ and least zone was observed in B. subtilis having a zone of $13 \pm 2.8 \mathrm{~mm}$.

Conclusion: This study showed that environmental factor such as sound and electromagnetic flux (EMF) could interfere with the physiology of bacteria including resistance/susceptibility to antibiotics. However, further investigation will be needed to understand full mechanisms of action of sound and electromagnetic field on bacteria.
\end{abstract}

Keywords: Audible sound, Electromagnetic flux, Typed strain culture, Music

\footnotetext{
*Correspondence: oluseungaruba@gmail.com

${ }^{1}$ Microbial Physiology and Biochemistry Research Laboratory,

Department of Microbiology, University of Ibadan, Ibadan, Nigeria

Full list of author information is available at the end of the article
}

\section{Background}

Several biophysical factors such as sound, electromagnetic radiation and electric current resulting from natural and anthropogenic activities have been reported to affect the growth and metabolism of various life forms 
(Moura et al. 2010; Movahedi et al. 2019). Audible sound has a wide distribution in natural world and nearly all life forms are surrounded by different kinds of sound and they also interact with these sounds and these have reportedly increase over the years (Sarvaiya and Kothari 2015; Kumar et al. 2018; Vitkauskaite and Grubliauskas 2018). Sound waves can create a mechanical stress to organisms exposed to them, and detection of such physical stress is a mechanism common to mounting a response against a broad range of environmental stresses. The possibility of the mechanical stress created by sound to trigger the expression of stress response genes in the organism exposed to such stress as also been reported (Walley and Dehesh 2010; Robinson et al. 2021).

In recent times, the misuse and abuse of antibiotics in therapy have thrown up facts of antibiotic susceptibility and resistance, respectively, with a correlation between them (Crofts et al. 2017; Li et al. 2020). Furthermore, the mechanisms of antibiotic activity on bacterial cells as well as cellular resistance to antibiotics are still subjects of active and intense scientific research (Abushaheena et al. 2020; Li et al. 2020). However, the biophysical factors that may affect antibiotic efficacy on microorganism have been underexplored and with the increasing human population and anthropogenic activities accompanied by gradual increase in the volume of sound waves and electromagnetic flux in our environment (Kumar et al. 2018; Vitkauskaite and Grubliauskas 2018; Movahedi et al. 2019; Robinson et al. 2021), these factors can have a great physiological effects on microbial growth and response to antibiotics, hence the need to properly investigate this phenomenon. In this study we report the effect of sound of different frequencies and decibels, and electromagnetic radiation on growth and antibiotic susceptibility of some selected Gram-positive and Gram-negative bacteria. This physiological study of bacterial response to sound waves and electromagnetic flux with respect to antibiotic susceptibility can shed more light on the interaction between biophysics and antibiotic efficacy and can also form a base-line physiological fact on the influence of gradually increasing sound pollution as well as electromagnetic flux proliferation on the emergence and persistence of antibiotics resistance.

\section{Methods}

\section{Microorganism}

Microorganisms used in this study were Gluconobacteroxydans ATCC 19,357, Rhodobacter sphaeroides ATCC 17,023, Citrobacter freundii ATCC 33,128, Yersina pestis ATCC 11,953, Bacillus subtilis ATCC 6633, Acetobacter aceti ATCC 15,973, Escherichia coli ATCC 25,922, Pseudomonas aeuriginosa ATCC 9027, Streptococcus pyogenes ATCC 19,613, Klebsiella pneumonia ATCC 25,955,
Staphylococcus aureus ATCC 25,923 and Serratia marcescens ATCC 14,766. The organisms were purchased from the American Type Culture Collection (ATCC), and they were stored at $4{ }^{\circ} \mathrm{C}$ on Nutrient Agar (Oxoid Uk) slants and sub-cultured fortnightly.

\section{Antibiotics susceptibility test pattern}

Antibiotic susceptibility of the organisms was performed by using eighteen to twenty four (18-22) hours old bacterial cultures using different antibiotics discs for both Gram-positive and Gram-negative organisms according to the Kirby-Bauer technique as described by Baker et al. (1980). The antibiotics used were: Ciprofloxacin $5 \mu \mathrm{g}$, Imipenem $10 \mu \mathrm{g}$, Ampicillin $10 \mu \mathrm{g}$, Ceftazidime $30 \mu \mathrm{g}$ and Tetracycline $30 \mu \mathrm{g}$ for Gram-negative bacteria while Pefloxacin $10 \mu \mathrm{g}$, Gentamycin $10 \mu \mathrm{g}$, Amplicillin + Cloxacillin $30 \mu \mathrm{g}$, Cefuroxime $20 \mu \mathrm{g}$, Amoxacillin $30 \mu \mathrm{g}$, Ceftriaxone $25 \mu \mathrm{g}$, Ciprofloxacin $10 \mu \mathrm{g}$, Streptomycin $30 \mu \mathrm{g}$, Co-trimoxazole $30 \mu \mathrm{g}$, and Erythromycin $10 \mu \mathrm{g}$ for Gram-positive bacteria, respectively. The discs were purchased from Oxoid chemicals (UK). The diameters of the inhibition or stimulation zone were measured after $24 \mathrm{~h}$, $48 \mathrm{~h}$ and $72 \mathrm{~h}$, respectively.

\section{Effect of sound of different frequencies on growth and antibiotic sensitivity pattern}

Exposure of the different organisms to different acoustic treatment was done in an anechoic chamber constructed locally as described by Guet al. (2010) using polyurethane foam of density $1 \mathrm{~g} / \mathrm{cm}^{3}$, wood and cotton fabric material in a cylindrical shape. The dimensions of the cylinder were 15 inches height, 12 inches diameter and had a rectangular orifice of dimension 5.6 inches by 6 inches plate 1 . Each of the test organism was inoculated into sterile nutrient broth and incubated for $24 \mathrm{~h}$, $48 \mathrm{~h}$ and $72 \mathrm{~h}$, respectively, and thereafter exposed to acoustic treatment (produced using a Bluetooth speaker which was remotely powered and placed directly above the inoculated nutrient broth-containing the test organisms) at different exposure time ranging between 24 and $72 \mathrm{~h}$, frequencies and decibel of $5000 \mathrm{~Hz} / 90 \mathrm{~dB}$ and $1125 \mathrm{~Hz} / 80 \mathrm{~dB}$ and growth observed on Jenway Spectrophotometer at $540 \mathrm{~nm}$. Sample without sound treatment served as control group. Incubation was done at $37 \pm 1{ }^{\circ} \mathrm{C}$ and growth was also checked on spectrophotometer after 24-72 h incubation duration (Guet al. 2010). Antibiotic susceptibility pattern was done before and after the acoustic exposure experiment as described earlier.

\section{Effect of electromagnetic field on growth and antibiotic susceptibility pattern}

To investigate the effect of electromagnetic on the growth and antibiotic susceptibility pattern of the different 


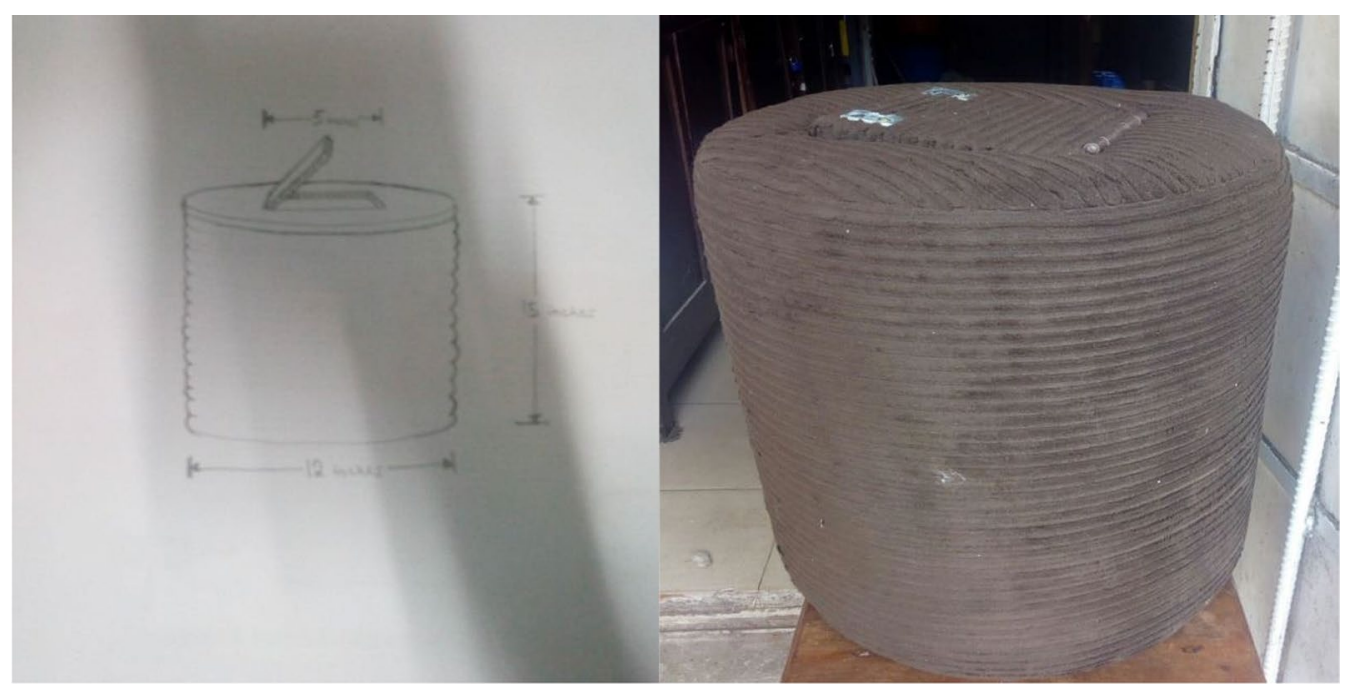

Plate 1 Schematic and Pictorial Representation of the Anechoic chamber used in this study

microorganism, actively growing bacterial cultures were inoculated into sterile nutrient broth and incubated at 37 $\mathrm{C}$ for $24 \mathrm{~h}$ and these were then exposed to low electromagnetic field as described by Garuba et al. (2020). The tubes of the bacteria to be exposed were placed in the middle of the coil by using supports to get a homogenous and higher magnetic field strength in which the ends of the solenoid were connected to variac fed from the mains $(220 \mathrm{~V})$. The magnetic field intensity was measured by means of hand held Gauss/Tesla meter, as the result proved its homogeneity among all the volume and was within $\pm 5 \%$ at the ends. Equal volumes of the bacterial culture were exposed for $15 \mathrm{~min}$ at their mid-to-late exponential phase (maximum rate of active growth) to the electromagnetic field at an intensity of $(5 \mathrm{mT})$, after exposure the growth rate was measured in a spectrophotometer and compared with the control (Mona et al. 2013).

\section{Statistical analysis}

Results obtained in this study were subjected to analysis of variance using ANOVA, and separation of means was carried out by Duncan's multiple range test (Duncan 1955).

\section{Results}

Twelve bacteria were subjected to two different acoustic treatments $(5000 \mathrm{~Hz} / 90 \mathrm{~dB}$ and $1125 \mathrm{~Hz} / 80 \mathrm{~dB})$ for duration of 24-72 h after which growth and antibiotic susceptibility pattern of each of the isolate measured. The results showed that all the bacteria culture at $5000 \mathrm{~Hz} / 90 \mathrm{~dB}$ had an increase growth $(\mathrm{p}<0.05)$ as compared with control except E. coli, K. pneumoniae and B. subtilis which decreased at $72 \mathrm{~h}$ acoustic exposure (Fig. 1). Similarly, exposure to sound of $1125 \mathrm{~Hz} / 80 \mathrm{~dB}$ for $72 \mathrm{~h}$ had varied effects on the growth of all the organisms investigated except for $S$. marcescens and $A$. acet $i$ which had a decline after $72 \mathrm{~h}$ exposure (Fig. 2).

Results of the antibiotics susceptibility pattern after the cultures were exposed to sound treatment at $5000 \mathrm{~Hz} / 90 \mathrm{~dB}$ and $1125 \mathrm{~Hz} / 80 \mathrm{~dB}$ is presented in Tables 1, 2, 3 and 4 . The results revealed that the exposure of $S$. marcescens to acoustic treatment at $5000 \mathrm{~Hz} / 90 \mathrm{~dB}$ showed increased resistance $(\mathrm{p}<0.05)$ to Ciprofloxacin at $24 \mathrm{~h}(30 \mathrm{~mm})$ and at $72 \mathrm{~h}(31 \mathrm{~mm})$ and maintained its resistance profile to tetracycline and Ampicillin throughout the exposure duration. $P$. aeruginosa became susceptible to Ampicillin after $72 \mathrm{~h}(11 \mathrm{~mm})$ having an initial resistance profile and treatment to Tetracycline showed resistance. G. oxydans which was initially susceptible became resistant to Ceftazidime after $24 \mathrm{~h}$ exposure while $A$. aceti became resistant to Ampicillin after exposure to treatment; $B$. subtilis showed increased susceptibility to Ampicillin after $24 \mathrm{~h}(27 \mathrm{~mm})$ at $\mathrm{p}<0.05$ and then became resistant after 48 and $72 \mathrm{~h}$ exposure, respectively, as shown in Tables 1 and 2. Similarly, an acoustic treatment of $1125 \mathrm{~Hz} / 80 \mathrm{~dB}, R$. sphaeroides showed increased susceptibility at $72 \mathrm{~h}(28 \mathrm{~mm})($ at $\mathrm{p}<0.05)$ to Ceftazidime and also to Imipenem $(34 \mathrm{~mm})$, respectively. A. aceti which had an initial susceptibility profile $(16 \mathrm{~mm})$ became resistant to Ampicillin at $48 \mathrm{~h}$ and $72 \mathrm{~h}$ exposure treatment, exposure of $S$. aureus to acoustic treatment showed increased resistance to Pefloxacin, Gentamycin, Ampicillin, Amoxacillin, Streptomycin all through the exposure duration, respectively, as shown in Tables 3 and 4 . 

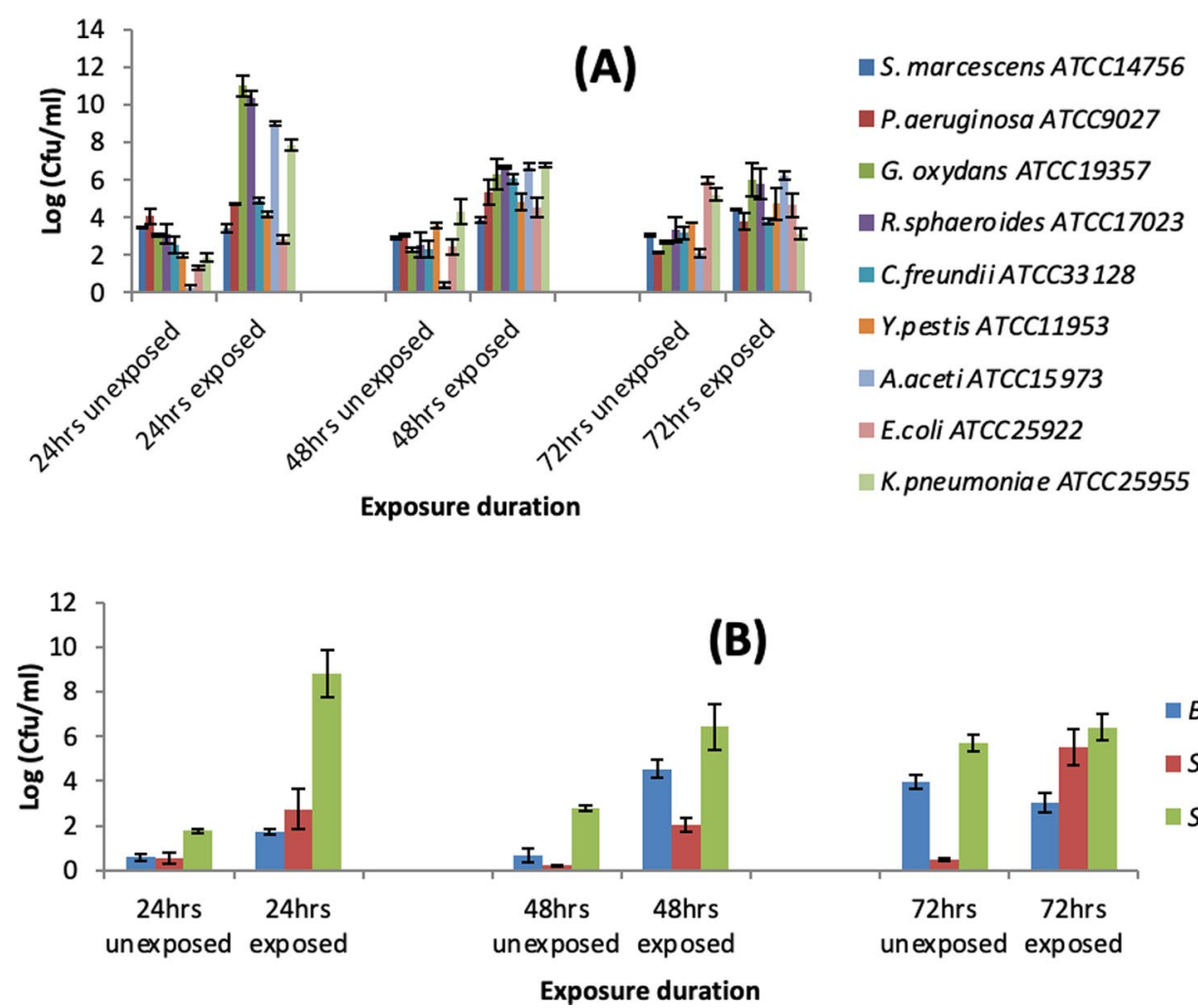

B.subtilis ATCC6633

S.pyogenes ATCC19613

S.aureus ATCC25923

Fig. 1 Growth rate of selected Gram-negative (a) and Gram-positive bacteria (b) after exposure to acoustic treatment at $5000 \mathrm{~Hz} / 90 \mathrm{~dB}$ compared with unexposed control

Exposure of the test organisms to electromagnetic flux of $5 \mathrm{mT}$ for $15 \mathrm{~min}$ was carried out and the growth alongside antibiotic susceptibility pattern investigated. The results presented in Tables 5 and 6 and Fig. 3 showed that $Y$. pestis, K. pneumonia and A. aceti had an increased growth $(\mathrm{p}<0.05)$ rate compared to control while other test bacteria had a decrease in growth compared to the control (Tables 5 and 6).

Electromagnetic exposure was also found to have varying effects on the antibiotic susceptibility pattern of the organisms under investigation. Exposure of G. oxydans showed an increase susceptibility to Tetracycline $(18 \mathrm{~mm}), Y$. pestis also showed an increase in susceptibility to Ceftazidime $(19 \mathrm{~mm})$ after electromagnetic treatment. B. subtilis became resistant to Ampicillin and $S$. aureus became resistant to Gentamycin, Ampicillin, Cefuroxime and Amoxacillin, respectively. S. pyogenes was observed to have an increase susceptibility to Pefloxacin $(20 \mathrm{~mm})$, Gentamycin $(17 \mathrm{~mm})$, Ampicillin $(13 \mathrm{~mm})$, Ceftriaxone (19 mm), Ciprofloxacin $(23 \mathrm{~mm})$, Streptomycin $(23 \mathrm{~mm})$, Cotrimoxazole $(24 \mathrm{~mm})$ and Erythromycin $(24 \mathrm{~mm})$, respectively, as shown in Table 5 and 6.

\section{Discussion}

In natural world, nearly all life forms are surrounded by different kinds of sound and electromagnetic fields from different sources (Dãnet 2005,) and there are few reports documenting the interactions between microorganisms, sound and electromagnetic fields (Gu et al. 2016; Movahedi et al. 2019; Robinson et al. 2021). However, the data provided by literature is not adequate in elucidating the physiological response to interactions between biological systems and these physical factors (that either occurs naturally or as a result of anthropogenic activities) (Guet al. 2010; Said-Salman et al. 2019). Furthermore, the use of physical signals such as sound waves, by the microorganisms for their intercellular communication has not received due attention (Chandra et al. 2018).

In this study, effect of low electromagnetic field and acoustic sound on the growth and antibiotics susceptibility pattern of twelve bacteria were investigated and some organisms were observed to respond to sound stimulation with a positive effect on growth, whereas sound waves had an inhibitory effect on the growth of some other microorganisms. This suggests that the action of sound on microbial growth showed obvious 


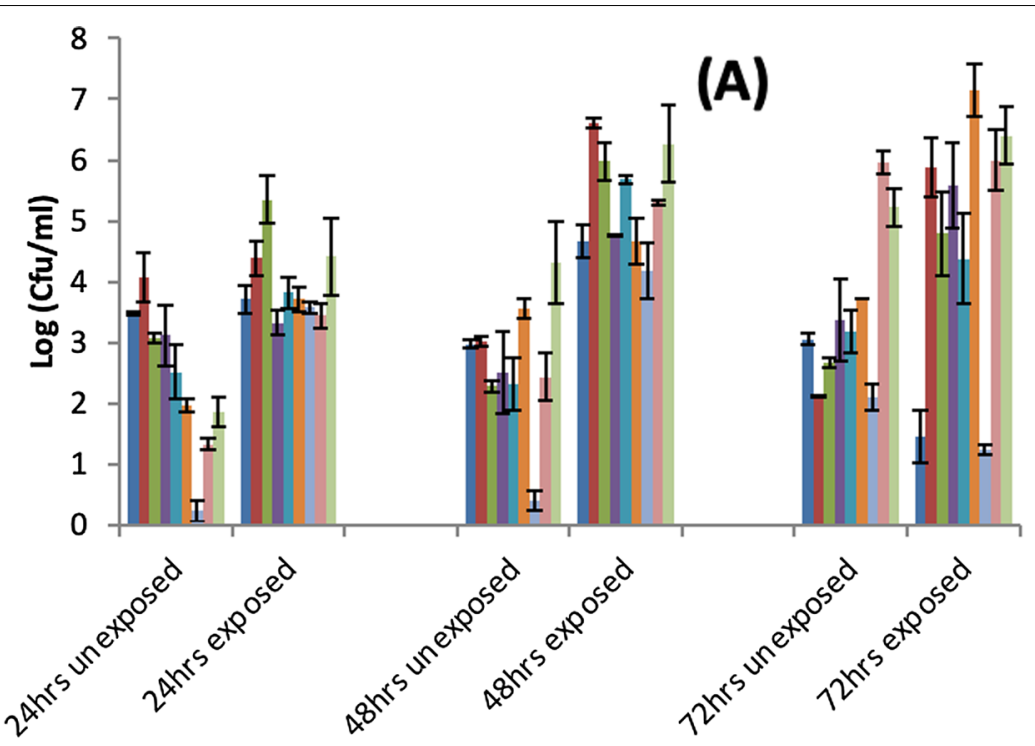

S.marcescens ATCC14756

- P. aeruginosa ATCC9027

G. oxydans ATCC19357

- R. sphaeroides ATCC17023

C. freundiiATCC33128

Y.pestis ATCC11953

A.acetiATCC15973

E.coli ATCC25922

K. pneumonia eATCC25955

Exposure duration

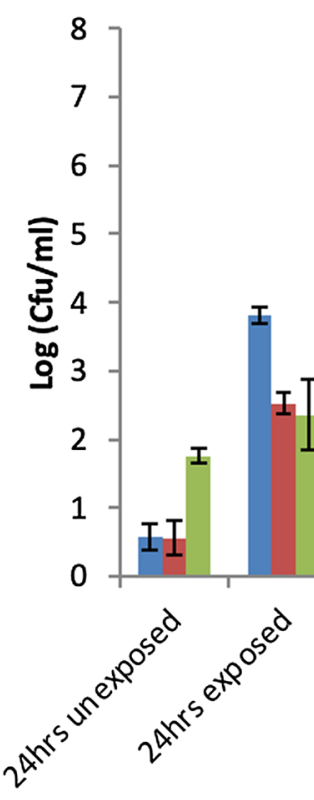

(B)

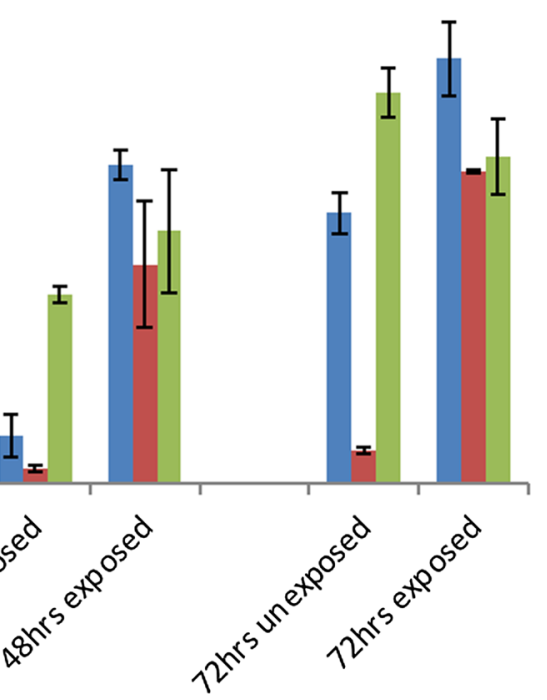

B. subtilis ATCC6633

S.pyogenes ATCC19613

S.aureus ATCC25923

\section{Exposure duration}

Fig. 2 Growth rate of selected Gram-negative (a) and Gram-positive bacteria (b) after exposure to acoustic treatment at $1125 \mathrm{~Hz} / 80 \mathrm{~dB}$ compared with unexposed control

frequency peculiarities. This peculiarity has been previously reported by Kothari et al. (2018). Similarly, Souvik et al. (2018) investigated the effect of three types of sound frequencies between $20 \mathrm{~Hz}$ and $20 \mathrm{kHz}$ on the growth and the antibiotic susceptibility pattern of $E$. coli. The results indicated that $E$. coli growth increased when exposed to sound frequency below $1 \mathrm{kHz}(432 \mathrm{~Hz})$ while at frequencies above $1 \mathrm{kHz}$, a poor growth was recorded. The altered growth rate observed has been suggested to be due to an altered movement of key molecules across the cell membrane of the organism as sound has been reported to increase membrane permeability in some microorganisms (Sarvaiya and Kothari 2015). The increased growth observed could be as a result of an adaptive response of the test bacteria to the sound exposure having an influence on microbial growth 
Table 1 Antibiotics susceptibility profile for selected Gram-negative bacteria as read in mean diameter of zones of inhibition (mm) after exposure to acoustic treatment at $5000 \mathrm{~Hz} / 90 \mathrm{~dB}$ compared with control

\begin{tabular}{|c|c|c|c|c|c|}
\hline Bacteria & CAZ & CIP & TET & IMP & AMP \\
\hline S. marcescens ATCC14756 & $\begin{array}{l}\text { a) } 26 \pm 1.4^{*} \\
\text { b) } 17 \pm 0 \\
\text { c) } 25 \pm 1.4 \\
\text { d) } 26 \pm 1.4\end{array}$ & $\begin{array}{l}\text { a) } 28 \pm 2.8 \\
\text { b) } 30 \pm 0.7 \\
\text { c) } 28 \pm 2.1 \\
\text { d) } 31 \pm 1.4\end{array}$ & $\begin{array}{l}\text { a) } R \\
\text { b) } R \\
\text { c) } R \\
\text { d) } R\end{array}$ & $\begin{array}{l}\text { a) } 26 \pm 1.4 \\
\text { b) } 19 \pm 2.1 \\
\text { c) } 24 \pm 1.4 \\
\text { d) } 25 \pm 0.7\end{array}$ & $\begin{array}{l}\text { a) } R \\
\text { b) } R \\
\text { c) } R \\
\text { d) } R\end{array}$ \\
\hline P. aeruginosa ATCC9027 & $\begin{array}{l}\text { a) } 26 \pm 1.4 \\
\text { b) } 23 \pm 0.7 \\
\text { c) } 20 \pm 1.4 \\
\text { d) } 22 \pm 0\end{array}$ & $\begin{array}{l}\text { a) } 34 \pm 3.5 \\
\text { b) } 29 \pm 1.4 \\
\text { c) } 21 \pm 0.7 \\
\text { d) } 22 \pm 3.5\end{array}$ & $\begin{array}{l}\text { a) } R \\
\text { b) } R \\
\text { c) } R \\
\text { d) } R\end{array}$ & $\begin{array}{l}\text { a) } 26 \pm 1.4 \\
\text { b) } 28 \pm 2.1 \\
\text { c) } 24 \pm 0.7 \\
\text { d) } 29 \pm 1.4\end{array}$ & $\begin{array}{l}\text { a) } R \\
\text { b) } R \\
\text { c) } R \\
\text { d) } 11 \pm 1.4\end{array}$ \\
\hline G. oxydans ATCC19357 & $\begin{array}{l}\text { a) } 27 \pm 2.8 \\
\text { b) } R \\
\text { c) } 29 \pm 1.4 \\
\text { d) } 19 \pm 4.9\end{array}$ & $\begin{array}{l}\text { a) } 26 \pm 0.7 \\
\text { b) } 27 \pm 3.5 \\
\text { c) } 21 \pm 2.1 \\
\text { d) } 21 \pm 0.7\end{array}$ & $\begin{array}{l}\text { a) } R \\
\text { b) } 17 \pm 2.8 \\
\text { c) } 12 \pm 0.7 \\
\text { d) } R\end{array}$ & $\begin{array}{l}\text { a) } 26 \pm 1.4 \\
\text { b) } 27 \pm 2.8 \\
\text { c) } 24 \pm 2.8 \\
\text { d) } 28 \pm 0.7\end{array}$ & $\begin{array}{l}\text { a) } 23 \pm 0.7 \\
\text { b) } 28 \pm 0.7 \\
\text { c) } 14 \pm 2.1 \\
\text { d) } R\end{array}$ \\
\hline R. sphaeroides ATCC17023 & $\begin{array}{l}\text { a) } 24 \pm 0.7 \\
\text { b) } 16 \pm 0 \\
\text { c) } R \\
\text { d) } 14 \pm 5.7\end{array}$ & $\begin{array}{l}\text { a) } 24 \pm 0.7 \\
\text { b) } 15 \pm 0 \\
\text { c) } 20 \pm 2.1 \\
\text { d) } 18 \pm 2.1\end{array}$ & $\begin{array}{l}\text { a) } R \\
\text { b) } R \\
\text { c) } 25 \pm 0.7 \\
\text { d) } R\end{array}$ & $\begin{array}{l}\text { a) } R \\
\text { b) } 19 \pm 0.7 \\
\text { c) } 21 \pm 0.7 \\
\text { d) } 23 \pm 5.7\end{array}$ & $\begin{array}{l}\text { a) } R \\
\text { b) } R \\
\text { c) } R \\
\text { d) } R\end{array}$ \\
\hline C. freundii ATCC33128 & $\begin{array}{l}\text { a) } 14 \pm 0.7 \\
\text { b) } 31 \pm 6.4 \\
\text { c) } 16 \pm 4.2 \\
\text { d) } 14 \pm 2.1\end{array}$ & $\begin{array}{l}\text { a) } 26 \pm 0.7 \\
\text { b) } 22 \pm 5.7 \\
\text { c) } 16 \pm 2.1 \\
\text { d) } 19 \pm 7.1\end{array}$ & $\begin{array}{l}\text { a) } 25 \pm 0 \\
\text { b) } 12 \pm 2.8 \\
\text { c) } 15 \pm 2.1 \\
\text { d) } 20 \pm 2.1\end{array}$ & $\begin{array}{l}\text { a) } 29 \pm 4.2 \\
\text { b) } 30 \pm 0.7 \\
\text { c) } 28 \pm 2.1 \\
\text { d) } 32 \pm 0.7\end{array}$ & $\begin{array}{l}\text { a) } 30 \pm 2.1 \\
\text { b) } 21 \pm 9.2 \\
\text { c) } R \\
\text { d) } R\end{array}$ \\
\hline Y. pestis ATCC11953 & $\begin{array}{l}\text { a) } 13 \pm 1.4 \\
\text { b) } 13 \pm 2.1 \\
\text { c) } 15 \pm 0.7 \\
\text { d) } 13 \pm 1.4\end{array}$ & $\begin{array}{l}\text { a) } 25 \pm 0 \\
\text { b) } 20 \pm 0.7 \\
\text { c) } 22 \pm 0.7 \\
\text { d) } 21 \pm 0.7\end{array}$ & $\begin{array}{l}\text { a) } R \\
\text { b) } R \\
\text { c) } R \\
\text { d) } R\end{array}$ & $\begin{array}{l}\text { a) } 23 \pm 1.4 \\
\text { b) } 18 \pm 5.7 \\
\text { c) } 24 \pm 1.4 \\
\text { d) } 25 \pm 0\end{array}$ & $\begin{array}{l}\text { a) } 15 \pm 0 \\
\text { b) } R \\
\text { c) } 13 \pm 0.7 \\
\text { d) } 21 \pm 2.1\end{array}$ \\
\hline K. pneumoniae ATCC25923 & $\begin{array}{l}\text { a) } 26 \pm 1.4 \\
\text { b) } 26 \pm 0 \\
\text { c) } 26 \pm 1.4 \\
\text { d) } 28 \pm 5.7\end{array}$ & $\begin{array}{l}\text { a) } 31 \pm 2.1 \\
\text { b) } 20 \pm 1.4 \\
\text { c) } 23 \pm 3.5 \\
\text { d) } 18 \pm 1.4\end{array}$ & $\begin{array}{l}\text { a) } R \\
\text { b) } 16 \pm 12.7 \\
\text { c) } 17 \pm 4.2 \\
\text { d) } R\end{array}$ & $\begin{array}{l}\text { a) } 22 \pm 2.8 \\
\text { b) } R \\
\text { c) } 25 \pm 4.9 \\
\text { d) } 31 \pm 1.4\end{array}$ & $\begin{array}{l}\text { a) } 24 \pm 0.7 \\
\text { b) } 21 \pm 1.4 \\
\text { c) } 14 \pm 6.4 \\
\text { d) } R\end{array}$ \\
\hline A. aceti ATCC15973 & $\begin{array}{l}\text { a) } 19 \pm 4.2 \\
\text { b) } 21 \pm 2.8 \\
\text { c) } 23 \pm 2.8 \\
\text { d) } 27 \pm 1.4\end{array}$ & $\begin{array}{l}\text { a) } 21 \pm 9.9 \\
\text { b) } 19 \pm 1.4 \\
\text { c) } 19 \pm 0 \\
\text { d) } 21 \pm 2.8\end{array}$ & $\begin{array}{l}\text { a) } 13 \pm 4.2 \\
\text { b) } 15 \pm 9.2 \\
\text { c) } 13 \pm 1.4 \\
\text { d) } 17 \pm 0.7\end{array}$ & $\begin{array}{l}\text { a) } 17 \pm 1.4 \\
\text { b) } 30 \pm 2.4 \\
\text { c) } 26 \pm 0.7 \\
\text { d) } 28 \pm 0\end{array}$ & $\begin{array}{l}\text { a) } 16 \pm 4.2 \\
\text { b) } R \\
\text { c) } R \\
\text { d) } R\end{array}$ \\
\hline E. coli ATCC25922 & $\begin{array}{l}\text { a) } 29 \pm 1.4 \\
\text { b) } 20 \pm 1.4 \\
\text { c) } R \\
\text { d) } 15 \pm 1.4\end{array}$ & $\begin{array}{l}\text { a) } 22 \pm 1.4 \\
\text { b) } 21 \pm 0.7 \\
\text { c) } 18 \pm 3.5 \\
\text { d) } 24 \pm 0.7\end{array}$ & $\begin{array}{l}\text { a) } 19 \pm 0 \\
\text { b) } R \\
\text { c) } 14 \pm 7.8 \\
\text { d) } R\end{array}$ & $\begin{array}{l}\text { a) } 33 \pm 4.2 \\
\text { b) } 30 \pm 0.7 \\
\text { c) } 30 \pm 2.1 \\
\text { d) } 29 \pm 1.4\end{array}$ & $\begin{array}{l}\text { a) } 26 \pm 1.4 \\
\text { b) } R \\
\text { c) } R \\
\text { d) } R\end{array}$ \\
\hline
\end{tabular}

a) Control b) $24 \mathrm{~h} \mathrm{c)} 48 \mathrm{~h}$ d) $72 \mathrm{~h} \mathrm{R}$-Resistance CAZ-Ceftazidime CIP_Ciprofloxacin

TET-Tetracycline IMP_Imipenem AMP_Ampicillin *Data are mean of replicate \pm Standard deviation

due to increase in membrane permeability while reduced growth observed could be as a result of mechanical stress created by the sound exposure after a long duration which could trigger the stress response of the bacteria hence resulting in the death of the organisms (Shah et al. 2016; Sarvaiya and Kothari, 2017; Kothari et al. 2018). This is because sound waves have been reported to travel through any medium giving rise to sonic vibrations which are thought to be sensed by the test microbial population through mechanosensory receptors (which act as safety valves against osmotic shock, and in higher organisms they participate in sensing touch and sound waves) (Kung et al. 2010; Ward et al. 2014; Shah et al. 2016). The microbial population may then modulate its behaviour in accordance to the magnitude and duration of the sonic vibration by generating appropriate physiological responses (Martinac 2012) such as opening of mechanosenstive ion channels which in turn affects movement of certain key ions across the cell membrane, ultimately resulting in an altered pattern of growth and metabolism (Vitkauskaite and Grubliauskas 2018). Antibiotic susceptibility pattern of the bacteria investigate in this study was also found to vary considerably with some of the becoming more susceptible while other became resistant as previously reported (Sarvaiya and Kothari 2017; Souvik et al. 2018). The altered antibiotic pattern of antibiotic sensitivity by the test organisms has been suggested to result from an altered transport (increased uptake) of the antibiotic used through the cell membrane via the mechanosensitive channel which responds to mechanical stress by 


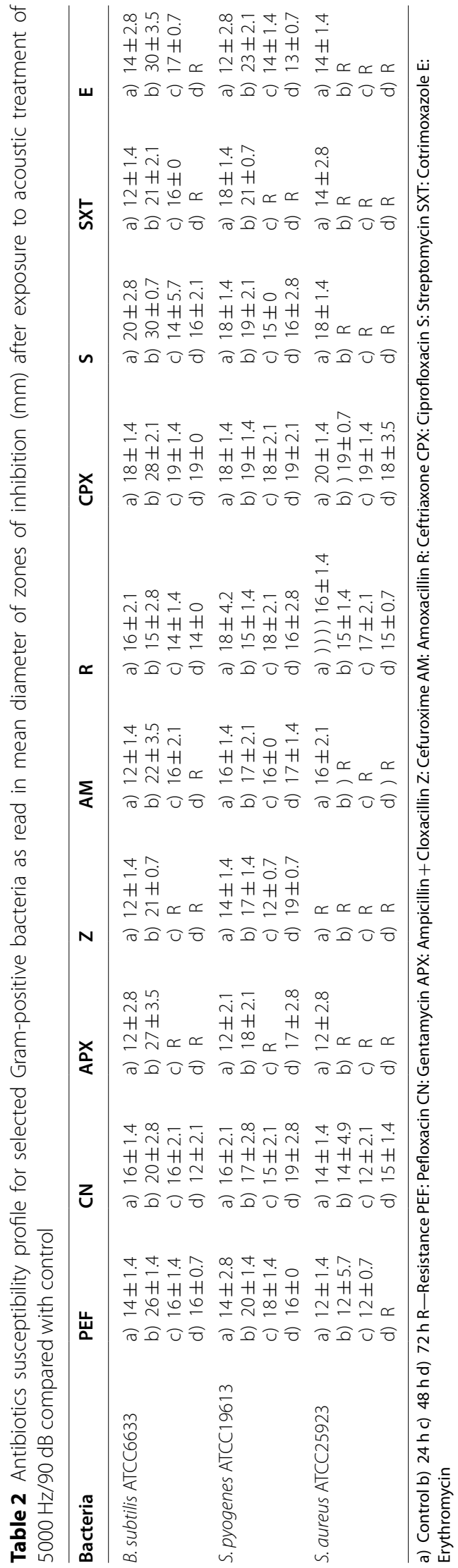


Table 3 Antibiotics susceptibility profile for selected Gram-negative bacteria as read in mean diameter of zones of inhibition (mm) after exposure to acoustic treatment at $1125 \mathrm{~Hz} / 80 \mathrm{~dB}$ compared with control

\begin{tabular}{|c|c|c|c|c|c|c|}
\hline Bacteria & Exposure & CAZ & CIP & TET & IMP & AMP \\
\hline S. marcescens ATCC14756 & $\begin{array}{l}\text { a) Control } \\
\text { b) } 24 \mathrm{~h} \\
\text { c) } 48 \mathrm{~h} \\
\text { d) } 72 \mathrm{~h}\end{array}$ & $\begin{array}{l}26 \pm 1.4 \\
23 \pm 1.4 \\
23 \pm 0 \\
20 \pm 9.2\end{array}$ & $\begin{array}{l}28 \pm 2.8 \\
21 \pm 1.4 \\
29 \pm 2.8 \\
25 \pm 0.7\end{array}$ & $\begin{array}{l}R \\
R \\
R \\
12 \pm 2.1\end{array}$ & $\begin{array}{l}2.6 \pm 1.4 \\
29 \pm 1.4 \\
32 \pm 1.4 \\
26 \pm 5.7\end{array}$ & $\begin{array}{l}R \\
R \\
R \\
19 \pm 4.2\end{array}$ \\
\hline $\begin{array}{l}\text { P. aeruginosa } \\
\text { ATCC9027 }\end{array}$ & $\begin{array}{l}\text { a) Control } \\
\text { b) } 24 \mathrm{~h} \\
\text { c) } 48 \mathrm{~h} \\
\text { d) } 72 \mathrm{~h}\end{array}$ & $\begin{array}{l}\text { a) } 26 \pm 1.4 \\
\text { b) } 20 \pm 9.2 \\
\text { c) } 26 \pm 2.1 \\
\text { d) } 30 \pm 0.7\end{array}$ & $\begin{array}{l}\text { a) } 34 \pm 3.5 \\
\text { b) } 23 \pm 3.5 \\
\text { c) } 22 \pm 0 \\
\text { d) } 27 \pm 1.4\end{array}$ & $\begin{array}{l}\text { a) } R \\
\text { b) } R \\
\text { c) } 27 \pm 2.8 \\
\text { d) } R\end{array}$ & $\begin{array}{l}\text { a) } 26 \pm 2.8 \\
\text { b) } 17 \pm 4.2 \\
\text { c) } R \\
\text { d) } 31 \pm 4.9\end{array}$ & $\begin{array}{l}\text { a) } R \\
\text { b) } R \\
\text { c) } R \\
\text { d) } R\end{array}$ \\
\hline $\begin{array}{l}\text { G. oxydans } \\
\text { ATCC19357 }\end{array}$ & $\begin{array}{l}\text { a) Control } \\
\text { b) } 24 \mathrm{~h} \\
\text { c) } 48 \mathrm{~h} \\
\text { d) } 72 \mathrm{~h}\end{array}$ & $\begin{array}{l}\text { a) } 27 \pm 2.8 \\
\text { b) } 20 \pm 0 \\
\text { c) } 14 \pm 2.1 \\
\text { d) } R\end{array}$ & $\begin{array}{l}\text { a) } 26 \pm 0.7 \\
\text { b) } 17 \pm 2.1 \\
\text { c) } 19 \pm 1.4 \\
\text { d) } 22 \pm 0.7\end{array}$ & $\begin{array}{l}\text { a) } R \\
\text { b) } R \\
\text { c) } 26 \pm 2.8 \\
\text { d) } R\end{array}$ & $\begin{array}{l}\text { a) } 26 \pm 1.4 \\
\text { b) } 24 \pm 3.5 \\
\text { c) } R \\
\text { d) } 18 \pm 13.4\end{array}$ & $\begin{array}{l}\text { a) } 23 \pm 0.7 \\
\text { b) } R \\
\text { c) } 18 \pm 0 \\
\text { d) } R\end{array}$ \\
\hline $\begin{array}{l}\text { R. sphaeroides } \\
\text { ATCC17023 }\end{array}$ & $\begin{array}{l}\text { a) Control } \\
\text { b) } 24 \mathrm{~h} \\
\text { c) } 48 \mathrm{~h} \\
\text { d) } 72 \mathrm{~h}\end{array}$ & $\begin{array}{l}\text { a) } 24 \pm 0.7 \\
\text { b) } 19 \pm 0.7 \\
\text { c) } 24 \pm 1.4 \\
\text { d) } 28 \pm 0.7\end{array}$ & $\begin{array}{l}\text { a) } 24 \pm 0.7 \\
\text { b) } 23 \pm 0 \\
\text { c) } 24 \pm 3.5 \\
\text { d) } 23 \pm 3.5\end{array}$ & $\begin{array}{l}\text { a) } R \\
\text { b) } R \\
\text { c) } 28 \pm 0.7 \\
\text { d) } R\end{array}$ & $\begin{array}{l}\text { a) } R \\
\text { b) } 23 \pm 3.5 \\
\text { c) } R \\
\text { d) } 34 \pm 0.7\end{array}$ & $\begin{array}{l}\text { a) } R \\
\text { b) } R \\
\text { c) } R \\
\text { d) } R\end{array}$ \\
\hline $\begin{array}{l}\text { C. freundii } \\
\text { ATCC } 33128\end{array}$ & $\begin{array}{l}\text { a) Control } \\
\text { b) } 24 \mathrm{~h} \\
\text { c) } 48 \mathrm{~h} \\
\text { d) } 72 \mathrm{~h}\end{array}$ & $\begin{array}{l}\text { a) } 14 \pm 0.7 \\
\text { b) } 14 \pm 0 \\
\text { c) } 20 \pm 7.1 \\
\text { d) } 30 \pm 0\end{array}$ & $\begin{array}{l}\text { a) } 26 \pm 0.7 \\
\text { b) } 24 \pm 0.7 \\
\text { c) } 31 \pm 2.1 \\
\text { d) } 30 \pm 1.4\end{array}$ & $\begin{array}{l}\text { a) } 25 \pm 0 \\
\text { b) } R \\
\text { c) } 12 \pm 0.7 \\
\text { d) } 11 \pm 1.4\end{array}$ & $\begin{array}{l}\text { a) } 29 \pm 4.2 \\
\text { b) } 25 \pm 0.7 \\
\text { c) } R \\
\text { d) } 30 \pm 0\end{array}$ & $\begin{array}{l}\text { a) } 30 \pm 2.1 \\
\text { b) } R \\
\text { C) } R \\
\text { d) } R\end{array}$ \\
\hline $\begin{array}{l}\text { Y. pestis } \\
\text { ATCC11953 }\end{array}$ & $\begin{array}{l}\text { a) Control } \\
\text { b) } 24 \mathrm{~h} \\
\text { c) } 48 \mathrm{~h} \\
\text { d) } 72 \mathrm{~h}\end{array}$ & $\begin{array}{l}\text { a) } 13 \pm 1.4 \\
\text { b) } 23 \pm 0.7 \\
\text { c) } 18 \pm 0 \\
\text { d) } 16 \pm 2.8\end{array}$ & $\begin{array}{l}\text { a) } 23 \pm 0 \\
\text { b) } 22 \pm 2.1 \\
\text { c) } 20 \pm 0.7 \\
\text { d) } 18 \pm 2.8\end{array}$ & $\begin{array}{l}\text { a) } R \\
\text { b) } R \\
\text { c) } R \\
\text { d) } R\end{array}$ & $\begin{array}{l}\text { a) } 23 \pm 1.4 \\
\text { b) } 20 \pm 4.9 \\
\text { c) } 26 \pm 0.7 \\
\text { d) } 23 \pm 0.7\end{array}$ & $\begin{array}{l}\text { a) } 15 \pm 0 \\
\text { b) } 14 \pm 2.1 \\
\text { c) } 15 \pm 0.7 \\
\text { d) } 17 \pm 1.4\end{array}$ \\
\hline $\begin{array}{l}\text { K. pneumoniae } \\
\text { ATCC25955 }\end{array}$ & $\begin{array}{l}\text { a) Control } \\
\text { b) } 24 \mathrm{~h} \\
\text { c) } 48 \mathrm{~h} \\
\text { d) } 72 \mathrm{~h}\end{array}$ & $\begin{array}{l}\text { a) } 26 \pm 1.4 \\
\text { b) } 27 \pm 1.4 \\
\text { c) } 26 \pm 0.7 \\
\text { d) } 26 \pm 2.8\end{array}$ & $\begin{array}{l}\text { a) } 31 \pm 2.1 \\
\text { b) } 21 \pm 1.4 \\
\text { c) } 23 \pm 0.7 \\
\text { d) } 25 \pm 3.5\end{array}$ & $\begin{array}{l}\text { a) } R \\
\text { b) } R \\
\text { c) } 25 \pm 5.7 \\
\text { d) } R\end{array}$ & $\begin{array}{l}\text { a) } 22 \pm 2.8 \\
\text { b) } 30 \pm 0.7 \\
\text { c) } R \\
\text { d) } 31 \pm 0.7\end{array}$ & $\begin{array}{l}\text { a) } 24 \pm 0.7 \\
\text { b) } R \\
\text { c) } R \\
\text { d) } R\end{array}$ \\
\hline $\begin{array}{l}\text { A.aceti } \\
\text { ATCC15973 }\end{array}$ & $\begin{array}{l}\text { a) Control } \\
\text { b) } 24 \mathrm{~h} \\
\text { c) } 48 \mathrm{~h} \\
\text { d) } 72 \mathrm{~h}\end{array}$ & $\begin{array}{l}\text { a) } 19 \pm 4.2 \\
\text { b) } 26 \pm 0 \\
\text { c) } 14 \pm 4.2 \\
\text { d) } 19 \pm 0.7\end{array}$ & $\begin{array}{l}\text { a) } 21 \pm 9.9 \\
\text { b) } 19 \pm 2.1 \\
\text { c) } 28 \pm 4.9 \\
\text { d) } 20 \pm 0\end{array}$ & $\begin{array}{l}\text { a) } 13 \pm 4.2 \\
\text { b) } R \\
\text { c) } 21 \pm 14.1 \\
\text { d) } 10 \pm 0\end{array}$ & $\begin{array}{l}\text { a) } 17 \pm 1.4 \\
\text { b) } 34 \pm 7.1 \\
\text { c) } 22 \pm 2.8 \\
\text { d) } 27 \pm 1.4\end{array}$ & $\begin{array}{l}\text { a) } 16 \pm 4.2 \\
\text { b) } 13 \pm 1.4 \\
\text { c) } R \\
\text { d) } R\end{array}$ \\
\hline $\begin{array}{l}\text { E. coli } \\
\text { ATCC25922 }\end{array}$ & $\begin{array}{l}\text { a) Control } \\
\text { b) } 24 \mathrm{~h} \\
\text { c) } 48 \mathrm{~h} \\
\text { d) } 72 \mathrm{~h}\end{array}$ & $\begin{array}{ll}\text { a) } & 29 \pm 1.4 \\
\text { b) } & 14 \pm 1.4 \\
\text { c) } & 17 \pm 0.7 \\
\text { d) } & 18 \pm 1.4\end{array}$ & $\begin{array}{l}\text { a) } 22 \pm 1.4 \\
\text { b) } 20 \pm 0.7 \\
\text { c) } 21 \pm 1.4 \\
\text { d) } 21 \pm 0.7\end{array}$ & $\begin{array}{l}\text { a) } 19 \pm 0 \\
\text { b) } R \\
\text { c) } 20 \pm 4.2 \\
\text { d) } R\end{array}$ & $\begin{array}{l}\text { a) } 33 \pm 4.2 \\
\text { b) } 20 \pm 0 \\
\text { c) } R \\
\text { d) } 27 \pm 1.4\end{array}$ & $\begin{array}{l}\text { a) } 26 \pm 1.4 \\
\text { b) } R \\
\text { c) } R \\
\text { d) } R\end{array}$ \\
\hline
\end{tabular}

a) —Control b) - $24 \mathrm{~h}$ c) $-48 \mathrm{~h}$ d) $-72 \mathrm{~h} \mathrm{R}$-Resistance CAZ—Ceftazidime CIP—Ciprofloxacin TET—Tetracycline IMP—Imipenem AMP—Ampicillin *Data are mean of replicate \pm S.D

changing its conformation between an open and closed state and this response is dependent on the frequency of sound, type of microorganism and exposure duration (Sarvaiya and Kothari 2017).

Non-ionising, non-thermal, extremely low frequency and electromagnetic fields (ELF-EMF) have been reported to produce a number of biological effects during their interaction with biological systems (Belyaev et al. 2011; Nguyen et al. 2015; Taheri et al. 2017a; Movahedi et al. 2019). In this study, there exists a decrease in growth rate after exposure to electromagnetic field of $5 \mathrm{mT}$. This observation is in accordance with Inhan-Garipet al. (2011) who carried an experiment on three Gram-negative and three Gram-positive bacteria and observed a decrease in growth of all test cultures after exposure to electromagnetic flux. This reduced cell growth observed could be a result of increased level of reactive oxygen species prompted by exposure to electromagnetic field which may have affected macromolecular synthesis and caused protein injury, ultimately leading to a decrease in bacterial growth rate as suggested by Souvik et al. (2018)

After exposure to electromagnetic field, some of the bacteria were found to developed resistance to antibiotics they were previously susceptible to while some became more susceptible. Chiefly among the several factors affecting antimicrobial susceptibility are the physical properties of the magnetic field, such as frequency, duration of radiation, the structure of the bacterial cell wall and the nature of the peptidoglycan properties in the cell wall of the Gram-positive and Gram-negative bacteria (specifically with changes in the bacterial cell wall protein as suggested by Taheri et al. (2017a) and Said-Salman et al. (2019). Mechanisms of acquired susceptibility or acquired resistance 


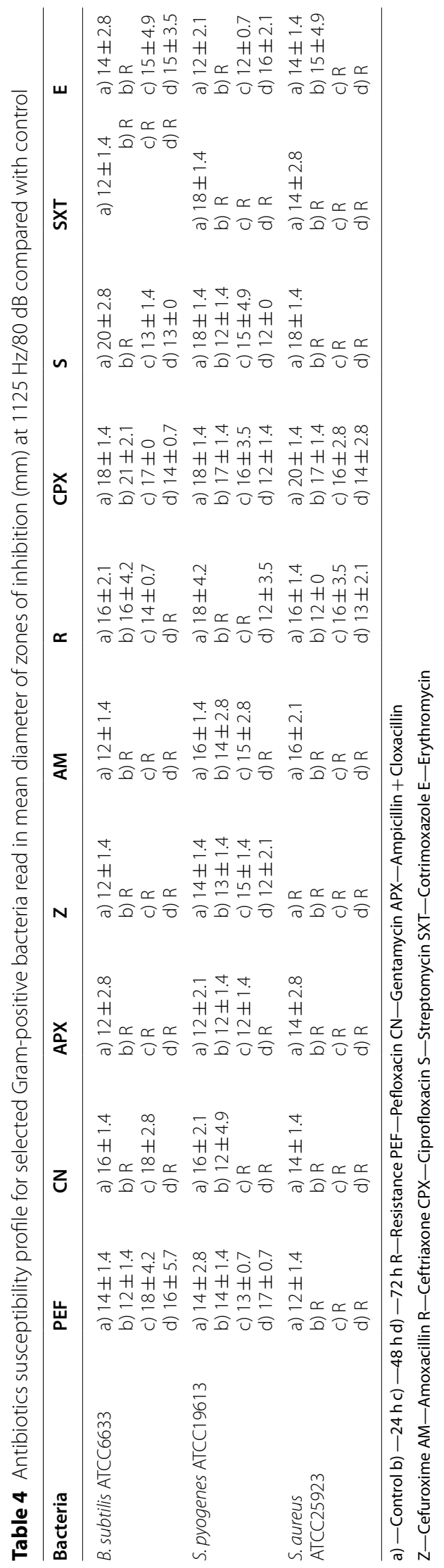


Table 5 Antibiotics susceptibility profile of selected Gram-negative bacteria as read in mean diameter of zones of inhibition (mm) after treatment to electromagnetic flux at $5 \mathrm{mT}$ intensity compared with control

\begin{tabular}{|c|c|c|c|c|c|}
\hline Bacteria culture & CAZ & CIP & TET & IMP & AMP \\
\hline S. marcescens ATCC14756 & $\begin{array}{l}\text { a) } 26 \pm 1.4 \\
\text { b) } 15 \pm 0.1\end{array}$ & $\begin{array}{l}\text { a) } 28 \pm 2.8 \\
\text { b) } 29 \pm 0.1\end{array}$ & $\begin{array}{l}\text { a) } R \\
\text { b) } R\end{array}$ & $\begin{array}{l}\text { a) } 26 \pm 1.4 \\
\text { b) } 21 \pm 2.8\end{array}$ & $\begin{array}{l}\text { a) } R \\
\text { b) } R\end{array}$ \\
\hline P. aeruginosa ATCC9027 & $\begin{array}{l}\text { a) } 26 \pm 1.4 \\
\text { b) } 16 \pm 0\end{array}$ & $\begin{array}{l}\text { a) } 34 \pm 3.5 \\
\text { b) } 26 \pm 8.5\end{array}$ & $\begin{array}{l}\text { a) } R \\
\text { b) } R\end{array}$ & $\begin{array}{l}\text { a) } 26 \pm 2.8 \\
\text { b) } 26 \pm 0\end{array}$ & $\begin{array}{l}\text { a) } R \\
\text { b) } R\end{array}$ \\
\hline G. oxydans ATCC19357 & $\begin{array}{l}\text { a) } 27 \pm 2.8 \\
\text { b) } 18 \pm 1.4\end{array}$ & $\begin{array}{l}\text { a) } 26 \pm 0.7 \\
\text { b) } 23 \pm 3.5\end{array}$ & $\begin{array}{l}\text { a) } R \\
\text { b) } 18 \pm 1.4\end{array}$ & $\begin{array}{l}\text { a) } 26 \pm 1.4 \\
\text { b) } 28 \pm 3.5\end{array}$ & $\begin{array}{l}\text { a) } 23 \pm 0.7 \\
\text { b) } R\end{array}$ \\
\hline R. sphaeroides ATCC17023 & $\begin{array}{l}\text { a) } 24 \pm 0.7 \\
\text { b) } R\end{array}$ & $\begin{array}{l}\text { a) } 24 \pm 0.7 \\
\text { b) } 24 \pm 0\end{array}$ & $\begin{array}{l}\text { a) } R \\
\text { b) } R\end{array}$ & $\begin{array}{l}\text { a) } R \\
\text { b) } 30 \pm 0.7\end{array}$ & $\begin{array}{l}\text { a) } R \\
\text { b) } R\end{array}$ \\
\hline C. freundii ATCC33128 & $\begin{array}{l}\text { a) } 14 \pm 0.7 \\
\text { b) } R\end{array}$ & $\begin{array}{l}\text { a) } 26 \pm 0.7 \\
\text { b) } 24 \pm 0.7\end{array}$ & $\begin{array}{l}\text { a) } 25 \pm 0 \\
\text { b) } R\end{array}$ & $\begin{array}{l}\text { a) } 29 \pm 4.2 \\
\text { b) } R\end{array}$ & $\begin{array}{l}\text { a) } 30 \pm 2.1 \\
\text { b) } R\end{array}$ \\
\hline Y.pestis ATCC11953 & $\begin{array}{l}\text { a) } R \\
\text { b) } 19 \pm 1.4\end{array}$ & $\begin{array}{l}\text { a) } 25 \pm 0 \\
\text { b) } 19 \pm 1.4\end{array}$ & $\begin{array}{l}\text { a) } R \\
\text { b) } R\end{array}$ & $\begin{array}{l}\text { a) } 23 \pm 1.4 \\
\text { b) } 21 \pm 1.4\end{array}$ & $\begin{array}{l}\text { a) } 15 \pm 0 \\
\text { b) } R\end{array}$ \\
\hline K. pneumoniae ATCC25955 & $\begin{array}{l}\text { a) } 26 \pm 1.4 \\
\text { b) } 20 \pm 0\end{array}$ & $\begin{array}{l}\text { a) } 31 \pm 2.1 \\
\text { b) } 19 \pm 1.4\end{array}$ & $\begin{array}{l}\text { a) } R \\
\text { b) } R\end{array}$ & $\begin{array}{l}\text { a) } 22 \pm 2.8 \\
\text { b) } 23 \pm 1.4\end{array}$ & $\begin{array}{l}\text { a) } 24 \pm 0.7 \\
\text { b) } R\end{array}$ \\
\hline A. aceti ATCC15973 & $\begin{array}{l}\text { a) } 9 \pm 4.2 \\
\text { b) } R\end{array}$ & $\begin{array}{l}\text { a) } 21 \pm 9.9 \\
\text { b) } 16 \pm 0.7\end{array}$ & $\begin{array}{l}\text { a) } 13 \pm 4.2 \\
\text { b) } R\end{array}$ & $\begin{array}{l}\text { a) } 17 \pm 1.4 \\
\text { b) } 23 \pm 2.8\end{array}$ & $\begin{array}{l}\text { a) } 16 \pm 4.2 \\
\text { b) } 18 \pm 0.7\end{array}$ \\
\hline E. coli ATCC25922 & $\begin{array}{l}\text { a) } 29 \pm 1.4 \\
\text { b) } R\end{array}$ & $\begin{array}{l}\text { a) } 22 \pm 1.4 \\
\text { b) } 18 \pm 0.7\end{array}$ & $\begin{array}{l}\text { a) } 19 \pm 0 \\
\text { b) } 14 \pm 2.1\end{array}$ & $\begin{array}{l}\text { a) } 33 \pm 4.2 \\
\text { b) } R\end{array}$ & $\begin{array}{l}\text { a) } 26 \pm 1.4 \\
\text { b) } R\end{array}$ \\
\hline
\end{tabular}

a) - Control b) - 15 min R-Resistance CAZ_Ceftazidime CIP-Ciprofloxacin TET-Tetracycline IMP-Imipenem AMP-Ampicillin *Data are mean of replicate \pm Standard deviation

Table 6 Antibiotics susceptibility profile for selected Gram-positive bacteria as read in mean diameter of zones of inhibition (mm) after treatment to electromagnetic flux at $15 \mathrm{mT}$ intensity compared with control

\begin{tabular}{|c|c|c|c|c|c|c|c|c|c|c|}
\hline Bacteria & PEF & $\mathrm{CN}$ & APX & Z & $A M$ & $\mathbf{R}$ & CPX & $S$ & SXT & $\mathrm{E}$ \\
\hline C6633 & $\begin{array}{l}\text { a) } 14 \pm 1.4 \\
\text { b) } 17 \pm 3.5\end{array}$ & $\begin{array}{l}\text { a) } 16 \pm 1.4 \\
\text { b) } 12 \pm 2.1\end{array}$ & $\begin{array}{l}\text { a) } 12 \pm 2.8 \\
\text { b) } R\end{array}$ & $\begin{array}{l}\text { a) } 12 \pm 1.4 \\
\text { b) } 13 \pm 0.7\end{array}$ & $\begin{array}{l}\text { a) } 12 \pm 1.4 \\
\text { b) } 13 \pm 2.8\end{array}$ & $\begin{array}{l}\text { a) } 16 \pm 2.1 \\
\text { b) } 19 \pm 0.7\end{array}$ & $\begin{array}{l}\text { a) } 18 \pm 1.4 \\
\text { b) } 21 \pm 1.4\end{array}$ & $\begin{array}{l}\text { a) } 20 \pm 2.8 \\
\text { b) } 22 \pm 2.8\end{array}$ & $\begin{array}{l}\text { a) } 12 \pm 1.4 \\
\text { b) } 20 \pm 4.9\end{array}$ & $\begin{array}{l}\text { a) } 14 \pm 2.8 \\
\text { b) } 21 \pm 0.7\end{array}$ \\
\hline S. pyogenes ATCC19613 & $\begin{array}{l}\text { a) } 14 \pm 2.8 \\
\text { b) } 20 \pm 2.8\end{array}$ & $\begin{array}{l}\text { a) } 16 \pm 2.1 \\
\text { b) } 17 \pm 2.1\end{array}$ & $\begin{array}{l}\text { a) } 12 \pm 2.1 \\
\text { b) } 13 \pm 1.4\end{array}$ & $\begin{array}{l}\text { a) } 14 \pm 1.4 \\
\text { b) } 14 \pm 1.4\end{array}$ & $\begin{array}{l}\text { a) } 16 \pm 1.4 \\
\text { b) } 16 \pm 2.1\end{array}$ & $\begin{array}{l}\text { a) } 18 \pm 4.2 \\
\text { b) } 19 \pm 1.4\end{array}$ & $\begin{array}{l}\text { a) } 18 \pm 1.4 \\
\text { b) } 23 \pm 1.4\end{array}$ & $\begin{array}{l}\text { a) } 18 \pm 1.4 \\
\text { b) } 23 \pm 1.4\end{array}$ & $\begin{array}{l}\text { a) } 18 \pm 1.4 \\
\text { b) } 24 \pm 0\end{array}$ & $\begin{array}{l}\text { a) } 12 \pm 2.1 \\
\text { b) } 24 \pm 2.1\end{array}$ \\
\hline S. aureus ATCC25923 & $\begin{array}{l}\text { a) } 12 \pm 1.4 \\
\text { b) } 16 \pm 6.7\end{array}$ & $\begin{array}{l}\text { a) } 14 \pm 1.4 \\
\text { b) } R\end{array}$ & $\begin{array}{l}12 \pm 2.8 \\
\text { b) } R\end{array}$ & $\begin{array}{l}\text { a) } R \\
\text { b) } R\end{array}$ & $\begin{array}{l}\text { a) } 16 \pm 2.1 \\
\text { b) } R\end{array}$ & $\begin{array}{l}\text { a) } 16 \pm 1.4 \\
\text { b) } 18 \pm 3.5\end{array}$ & $\begin{array}{l}\text { a) } 20 \pm 1.4 \\
\text { b) } 20 \pm 0\end{array}$ & $\begin{array}{l}\text { a) } 18 \pm 1.4 \\
\text { b) } 18 \pm 2.8\end{array}$ & $\begin{array}{l}\text { a) } 14 \pm 2.8 \\
\text { b) } 14 \pm 0\end{array}$ & $\begin{array}{l}\text { a) } 14 \pm 1.4 \\
\text { b) } 17 \pm 4.2\end{array}$ \\
\hline
\end{tabular}

a) - Control b) - 24 h c) - 48 h d) - 72 h R-Resistance PEF-Pefloxacin, CN-Gentamycin, APX-Ampicillin + Cloxacillin, Z-Cefuroxime, AM-Amoxacillin, RCeftriaxone, CPX-Ciprofloxacin, S-Streptomycin, SXT-Cotrimoxazole, E-Erythromycin

(after exposure to EMF) are reported to be largely due to the importance of the membrane potential of the bacterial cell wall, these waves can have an effect on the antibiotic susceptibility (Torgomyan et al. 2011). Furthermore, increased antibiotic susceptibility may be due to the interaction of electromagnetic fields on the water molecules in the cell. An increase in the permeability of the bacteria which might make the ion channels to be kept open in the bacterial wall, efflux pumps and ion channels in the cell wall, could also be playing an important role in the absorption of antibiotics. Interaction between the electromagnetic waves and the molecules of the antibiotics (such interaction affecting the Hydrophilicity or hydrophobicity of the antibiotic, antibiotic activity and antibiotic molecule size) thereby altering the charge distribution on antibiotics molecule has also been reported to affect the transportation of antibiotics across the cell membrane (Taheri et al. 2017b).

So far, the process of antibiotic resistance (with accelerated increase through various anthropogenic activities) seems to proceed unabated. Results of this study can further help in understanding the various mechanisms of antibiotic resistance thereby adding valuable angles in the development of effective antibiotic therapy for treatment of infectious diseases. Based on findings in this work, the roles of mechanosensitive channels present on bacterial membranes with respect to permissively or selectively admitting antibiotic molecules crossing the membrane barriers can be further studied. The effects of electromagnetic flux and sound waves demonstrated in this study could be incorporated in the evaluation of mechanosensitive channels as pharmacological targets for novel antibiotics design. It has recently been identified that the antibiotic streptomycin can act as an unlocking agent of 

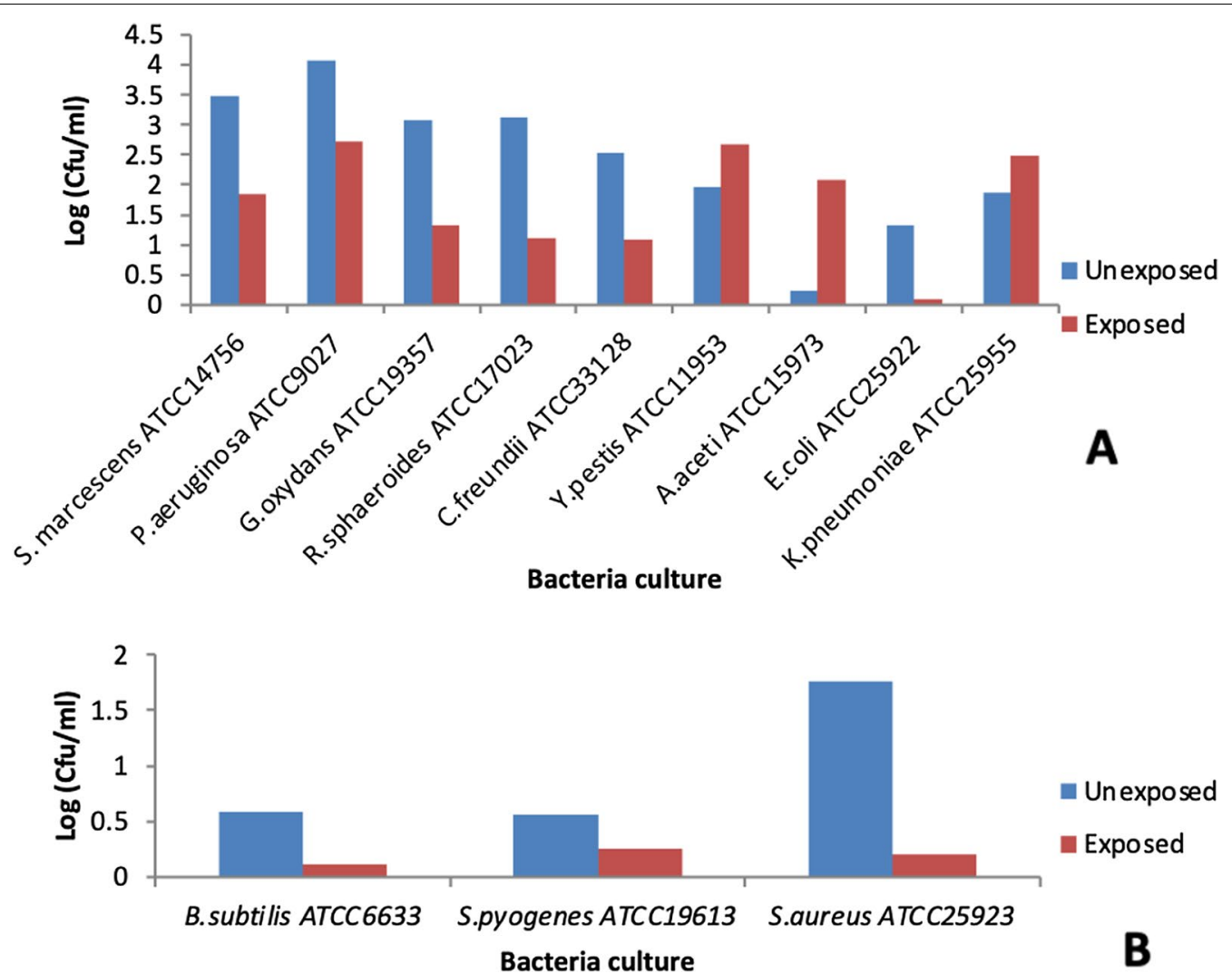

Fig. 3 Growth of selected Gram-negative bacteria (a) and Gram-positive bacteria (b) after treatment to electromagnetic flux of $5 \mathrm{mT}$ intensity after $24 \mathrm{~h}$ of incubation compared with unexposed control

large mechanosensitive channels in bacteria, as the drug uses the channels as a route of entry into the cytoplasm (Blount and Iscla 2020). Further research has also identified the potential promiscuity of mechanosensitive channels in some species in allowing access to multiple antibiotics into the cytoplasm depending on the size and vibrational effect (Wray et al. 2019).

Earlier reports have identified bacteria to possess potentials to respond specifically to vibrational patterns that induce ionic flow and electrical charges on the membrane which most likely are unique to each species (Norris and Hyland 1997). It was shown that soundinduced extracellular vibrations could be detected as unique acoustic signals that influence intracellular vibrations linked to the movements of intracellular organelles like molecular motors, cytoskeletons, and chromosomal package in a specific pattern (Reguera 2011). Electromagnetic flux has also been identified as particularly important in modulating microbial metabolism in a method that can be detrimental or beneficial to cellular proliferation depending on the specifics of the flux frequency and intensity (Beretta et al. 2019). Specific examples with respect to Pseudomonas and Enterobacter species showed that zero magnetic fields induced antibiotic resistance in these bacteria in a magnetosensitive pattern (Creanga et al. 2004). This clearly means that understanding of the exact electromagnetic and sound frequencies that control membrane mechanosensation and intracellular vibrations can be utilised in species-specific microbial responses to sound and electromagnetic flux. To consolidate on the effects of sound, there have been practical observations of acoustic frequencies emitted by a $B$. subtilis strain detected at a range of $8-43 \mathrm{kHz}$ (Matsuhashi et al. 1998), while a lower range of $0.9-1.6 \mathrm{kHz}$ was observed for Saccharomyces cerevisiae cells (Pelling et al. 2004). Apart from cellular vibrational patterns, it is understood that sound waves are propagated by mere vibration of particles within a medium (Reguera 2011), this therefore means that there can also be distinct sound patterns as observed within certain microbial ecological environments. These sound patterns could also be further studied as signals and frequencies and built upon to determine the specific responses of such bacteria to modulated sounds; a process that can be harnessed in designing therapeutics targeting sound-sensitivity in bacteria. 
With the exposition of this knowledge, it could then be easier to design adjuvants or small sized frequency specific agonists that can be used to complement the existing antibacterial drugs in a synergistic fashion against target bacteria, especially drug resistant phenotypes. Data generated in this work can also potentially enrich aspects of sonobiology and sonogenetics for further studies of microbial membrane and intracellular behaviour. Studies involving recently applied biomolecular ultrasound techniques have elucidated species-specific acoustic modulation of bacterial membranes and intramolecular materials for higher resolution imaging and characterisation of intracellular activities (Maresca et al. 2018). Results from this work could be further expounded upon to provide a basis for deeper experimentation on variable species behaviour for improvement into direct sonar scanning and imaging of bacteria in the future.

Industrially, the information from this study can be useful for strain improvement of microorganisms for enhanced production of desired microbial metabolites where applicable. This current study can also be applied in advancing the techniques of sonoporation in bacteria, as a combined application of electromagnetic pulses and sound can be specifically made to target viable cells. Pores formed on the cells can be sites of DNA entry in transformation experiments, as well as points for seepage of viable intracellular metabolites of molecules (Song et al. 2007). These data could also be a basis for design of more effective sonobiorecators (Chisti 2003) and electrobioreactors (Mohtasham et al. 2016) for bioprocess optimisation. Ultimately this new research area should open a new frontier for multidisciplinary work at the interfaces of molecular biology, biochemistry and biophysics especially bioacoustics and sonobiology.

Despite the proper evaluations of potential application of the data in this study, it is important to note that, specific experiments linking measured cell membrane mechanosensation in line with the permissiveness to antibiotics as well as growth patterns were not fully elucidated here. We however, have extrapolated potential reasons explaining the behaviour of the bacterial cells under the influence of sound vibrations and electromagnetic flux. In line with this it is therefore necessary to further investigate mechanosensory biology, along with transcriptome and/or metabolomic profiling of sound and low electromagnetic stimulated cultures using different microorganisms in other to provide meaningful understanding in the area of cellsound and cell-EMF interactions.

\section{Conclusions}

In this study, it has been shown that physical factors such as sound and electromagnetic flux (EMF) that abound in the environment interfere with the physiology of bacteria either by enhancing or inhibiting its growth as well as its response to antibiotics. This further point to the fact these factors can affect biomass accumulation and synthesis of intracellular molecules via a range of pathways, and that certain frequencies and amplitudes may favour some microbial species over others. This work also identifies the need for deeper investigations into the biomechanistic approaches microbial cells utilise in balancing membrane mechanosensitivity under the influence of sound and electromagnetic frequencies. The desired frequencies that could be applied in modulating cellular activities positively or negatively for applications in more efficient antimicrobial therapies and bioproduction processes are a major point of reference going forward.

\section{Abbreviations}

ATCC: American type culture collection; EMF: Electromagnetic field.

\section{Acknowledgements}

The authors are grateful to Profs N. N. Jibri and J. A. Adegoke of the Department of Physics, Faculty of Science, University of Ibadan for their technical assistance and contribution in the construction of the electromagnetic field apparatus and also to the technical staff of the Department of Microbiology, University of Ibadan for the Technical assistance provided during the period of this work

\section{Authors' contributions}

EOG and OMA designed the work. ANI-K collected the data and also prepared the manuscript together with EOG and OMA. EOG analysed some of the data. All authors read and approved the final manuscript.

Funding

No external source of funding was received for this work.

Availability of data and materials

The datasets used and/or analysed during the current study are available from the corresponding author on reasonable request.

\section{Declarations}

Ethics approval and consent to participate Not applicable.

Consent for publication

Not applicable.

\section{Competing interests}

The authors declare that they have no competing interests.

\section{Author details}

${ }^{1}$ Microbial Physiology and Biochemistry Research Laboratory, Department of Microbiology, University of Ibadan, Ibadan, Nigeria. ${ }^{2}$ Department of Microbiology, Modibbo Adama University of Technology, Yola, Nigeria.

Received: 23 September 2021 Accepted: 20 November 2021 Published online: 11 December 2021 


\section{References}

Abushaheena MA, Muzaheed Fatani AJ, Alosaimi M, Mansyd W, Georgef M, Acharya S, Rathod S, Divakar DD, Jhugroo C, Vellappally S, Khan AA, Shaik J, Jhugroo P (2020) Antimicrobial resistance, mechanisms and its clinical significance. Dis Mon 66:100971

BakerF CC, Misasi R, Deperdio R, Zimatore G (1980) Coducdomatic properties of human erythrocyte membranes: dependence on haematocrat and alkali metalions of the suspending medium. Euro Biophy 26:21-225

Belyaev I (2011) Toxicity and SOS-response to ELF magnetic fields and nalidixic acid in E. coli cells. Mutat Res 722:56-61. https://doi.org/10.1016/j.mrgen tox.2011.03.012

Beretta G, Mastorgio AF, Pedrali L, Saponaro S, Sezenna E (2019) The effects of electric, magnetic and electromagnetic fields on microorganisms in the perspective of bioremediation. Rev Environ Sci Biotechnol. https://doi. org/10.1007/s11157-018-09491-9

Blount P, Iscla I (2020) Life with bacterial mechanosensitive channels, from discovery to physiology to pharmacological target. Microbiol Mol Biol Rev 84(1):e00055-19. https://doi.org/10.1128/MMBR.00055-19

Chandra T, Lekha VS, Krishna TM (2018) Effect of music on growth and pigment production of brevibacterium sp. Int J Pharm Chem Bio Sci $8(1): 157-160$

Chisti Y (2003) Sonobioreactors: using ultrasound for enhanced microbial productivity. Trends Biotechnol 21(2):89-93

Creanga DE, Poiata A, Morairu W, Tupu P (2004) Zero-Magnetic field effect in pathogenic bacteria. J Mag Mag Mater 272-276:2442-2444. https://doi. org/10.1016/j.jmmm.2003.12.853

Crofts TS, Gasparrini AJ, Dantas G (2017) Next-generation approaches to understand and combat the antibiotic resistome. Nat Rev Microbiol $15: 422-434$

Dãnet AF (2005) Environmental pollution monitoring: pollution, analysis, legislation, quality assurance and managing. SC Pro Act Birotic SRL, Bucharest, pp 56-64

Duncan DB (1955) Multiple range and multiple F tests. Biometrics 11:1-42

Garuba EO, Obinna MA, Olaifa KW, Onilude AA (2020) Response Surface Methodology (RSM) and electromagnetic optimization of pigment production by Sporobolomyces sp S5 and Rhodotorula sp A21 in submerged fermentation. J BioSci Biotech 9(1):17-25

Gu SB, Wu Y, Li KW, Li SC, Ma SY, Wang QN, Wang R (2010) A pilot study of the effect of audible sound on the growth of Escherichia coli. Colloids Surf B Biointerfaces 78:367-371

Gu S, Zhang Y, Wu Y (2016) Effects of sound exposure on the growth and intracellular macromolecular synthesis of E. coli k12. PeerJ 4:e1920. https://doi. org/10.7717/peerj.1920

Inhan-Garip A, Aksu B, Akan Z, Akakin D, Ozaydin N, San T (2011) Effect of extremely low frequency electromagnetic fields on growth rate and morphology of bacteria. Int J Radiat Biol 87(12):1155-1161

Kothari V, Joshi C, Patel P, Mehta M, Dubey S, Mishra B, Sarvaiya N (2018) Influence of a mono-frequency sound on bacteria can be a function of the sound-level. Indian J Sci Technol. https://doi.org/10.17485/ijst/2018/ v11i4/111366

Kumar A, Kumar P, Mishra RK, Shukla A (2018) Study of air and noise pollution in mega cities of India. In: Singh VP, Yadav S, Yadava RN (eds) Environmental pollution. Springer, Berlin

Kung C, Martinac B, Sukharev S (2010) Mechanosensitive channels in microbes. Annu Rev Microbiol 64:313-329

Li L, Huang Q, Yin X, Zhang T (2020) Source tracking of antibiotic resistance genes in the environment: challenges, progress, and prospects. Water Res 185:116127. https://doi.org/10.1016/j.watres.2020.116127

Maresca D, Lakshmanan A, Abedi M, Bar-Zion A, Farhadi A, Lu GJ, Szablowski JO, Wu D, Yoo S, Sapiro MG (2018) Biomolecular ultrasound and sonogenetics. Annu Rev Chem Biomol Eng 9:229-252. https://doi.org/10.1146/ annurev-chembioeng-060817-084034

Martinac B (2012) Mechanosensitive ion channels: an evolutionary and scientific tour de force in mechanobiology. Channels 6(4):211-213. https://doi. org/10.4161/chan.22047

Matsuhashi M, Pankrushina AN, Takeuchi S, Ohshima H, Miyoi H, Endoh K Murayama K, Watanabe H, Endo S, Tobi M, Mano Y, Hyodo M, Kobayashi T, Kaneko T, Otani S, Yoshimura S, Harata A, Sawada T (1998) Production of sound waves by bacterial cells and the response of bacterial cells to sound. J Gen Appl Microbiol 44:49-55
Mohtasham P, Keshavarz-Moore E, Kale I, Keshavarz T (2016) Application of magnetic field for improvement of microbial productivity. Chem Eng Trans 49:43-48. https://doi.org/10.3303/CET1649008

Mona H, Ibraheim D, El-Din D (2013) 50 Hz frequency magnetic field effects on Pseudomonas aeruginosa and Bacillus subtilis bacteria. J Appl Phy 5:49-56

Moura A, Henriques I, Smalla K, Correia A (2010) Wastewater bacterial communities bring together broad-host range plasmids, integrons and a wide diversity of uncharacterized gene cassettes. Res Microbiol 161:58-66

Movahedi MM, Nouri F, Tavakoli Golpaygani A, Ataee L, Amani S, Taheri M (2019) Antibacterial susceptibility pattern of the Pseudomonas aeruginosa and Staphylococcus aureus after exposure to electromagnetic waves emitted from mobile phone simulator. J Biomed Phys Eng 9(6):637-646. https://doi.org/10.31661/jbpe.v0i0.1107

Nguyen TH, Shamis Y, Croft RJ, Wood A, McIntosh RL, Crawford RJ et al (2015) $18 \mathrm{GHz}$ electromagnetic field induces permeability of gram-positive cocci. Sci Rep 5:10980. https://doi.org/10.1038/srep10980

Norris V, Hyland GJ (1997) Do bacteria sing? Sonic intercellular communication between bacteria may reflect electromagnetic intracellular communication involving coherent collective vibrational modes that could integrate enzyme activities and gene expression. Mol Microbiol 24:879-880

Pelling AE, Sehati S, Gralla EB, Valentine JS, Gimzewski JK (2004) Local nanomechanical motion of the cell wall of Saccharomyces cerevisiae. Science 305:1147-1150

Reguera G (2011) When microbial conversations get physical. Trends Microbiol 19(3):105-113

Robinson JM, Cameron R, Paker B (2021) The effects of anthropogenic sound and artificial light exposure on microbiomes: ecological and public health implications. Front Ecol Evol 9:662588. https://doi.org/10.3389/ fevo.2021.662588

Said-Salman IH, Jebaii FA, Yusef HH, Moustafa ME (2019) Evaluation of wi-fi radiation efects on antibiotic susceptibility, metabolic activity and biofilm formation by Escherichia Coli 0157H7, Staphylococcus aureus and Staphylococcus epidermis. J Biomed Phys Eng 5:579-586. https://doi.org/ 10.31661/jbpe.v0i0.1106

Sarvaiya N, Kothari V (2017) Audible sound in form of music can influence microbial growth, metabolism and antibiotic susceptibility. J App Biotech Bioeng 2(6):212-219

Sarvaiya N, Kothari V (2015) Effect of audible sound in form of music on microbial growthand production of certain important metabolites. Microbiol 2:227-235

Shah A, Akansha R, Kothari V (2016) Sound stimulation can influence microbial growth and production of certain key metabolites. J Microbiol Biotechnol Food Sci 5(4):330

Song Y, Hahn T, Thompson IP, Mason TJ, Preston GM, Li G, Paniwnyk L, Huang WE (2007) Ultrasound-mediated DNA transfer for bacteria. Nucleic Acids Res 35(19):e129. https://doi.org/10.1093/nar/gkm710

Souvik B, Abhishek G, Ananya D, Anish P, Anjoom N, Bodhisattwa G (2018) Effect of different sound frequencies on the growth and antibiotic susceptibility of Escherichia coli. Int J CurrMicrobio Appl Sci 7(3):2319-7706

Taheri M, Moradi M, Mortazavi S, Mansouri S, Hatam G, Nouri F (2017a) Evaluation of the $900 \mathrm{MHz}$ radiofrequency radiation effects on the antimicrobial susceptibility and growth rate of Klebsiella pneumoniae. Shiraz E-Med J 18(3):e44946. https://doi.org/10.17795/semj44946

Taheri M, Mortazavi SMJ, Moradi M, Mansouri S, Hatam GR, Nouri F (2017b) Evaluation of the effect of radiofrequency radiation emitted from $\mathrm{Wi}-\mathrm{Fi}$ router and mobile phone simulator on the antibacterial susceptibility of pathogenic bacteria Listeria monocytogenes and Escherichia coli. Dose Response 15:1-8. https://doi.org/10.1177/1559325816688527

Torgomyan H, Tadevosyan H, Trchounian A (2011) Extremely high frequency electromagnetic irradiation in combination with antibiotics enhances antibacterial effects on Escherichia coli. Curr Microbiol 62:962-967. https://doi.org/10.1007/s00284-010-9811-2

Vitkauskaite G, Grubliauskas R (2018) Perforated sound-absorbing constructions acoustic performance test and noise modeling. Energy Procedia 147:288-294. https://doi.org/10.1016/j.egypro.2018.07.094

Walley JW, Dehesh K (2010) Molecular mechanisms regulating rapid stress signaling network in Arabidopsis. J Integ Plant Biol 4:354-359

Ward R, Pliotas C, Branigan E, Hacker C, Rasmussen A, Hagelueken G, Schiemann $O$ (2014) Probing the structure of the mechanosensitive channel of small conductance in lipid bilayers with pulsed electron-electron double 
resonance. Biophys J 106(4):834-842. https://doi.org/10.1016/j.bpj.2014. 01.008

Wray R, Wang J, Iscla I, Blount P (2019) Novel MscL agonists that allow multiple antibiotics cytoplasmic access activate the channel through a common binding site. PLoS ONE 15(1):e0228153. https://doi.org/10.1371/journal. pone. 0228153

\section{Publisher's Note}

Springer Nature remains neutral with regard to jurisdictional claims in published maps and institutional affiliations.

\section{Submit your manuscript to a SpringerOpen ${ }^{\circ}$ journal and benefit from:}

- Convenient online submission

- Rigorous peer review

- Open access: articles freely available online

- High visibility within the field

- Retaining the copyright to your article

Submit your next manuscript at $\boldsymbol{\nabla}$ springeropen.com 